This is the post peer-review accepted manuscript of:

A. Girolami, D. Brunelli and L. Benini, "Low-cost and distributed health monitoring system for critical buildings," 2017 IEEE Workshop on Environmental, Energy, and Structural Monitoring Systems (EESMS), Milan, 2017, pp. 1-6.

The published version is available online at:

https://doi.org/10.1109/EESMS.2017.8052686

(C) 2017 IEEE. Personal use of this material is permitted. Permission from IEEE must be obtained for all other uses, in any current or future media, including reprinting/republishing this material for advertising or promotional purposes, creating new collective works, for resale or redistribution to servers or lists, or reuse of any copyrighted component of this work in other works. 


\title{
Low-cost and distributed health monitoring system for critical buildings
}

\author{
Alberto Girolami ${ }^{1}$, Davide Brunelli ${ }^{2}$, Luca Benini ${ }^{1,3}$ \\ ${ }^{1}$ DEI, University of Bologna, 40136 Bologna, email: \{alberto.girolami2, luca.benini\}@unibo.it \\ ${ }^{2}$ DII, University of Trento, 38123 Trento, email: davide.brunelli@unitn.it \\ ${ }^{3}$ IIS, ETH Zurich, email: luca.benini@iis.ee.ethz.ch
}

\begin{abstract}
In this paper we present a low-cost distributed embedded system for Structural Health Monitoring (SHM) that uses very cost-effective MEMS accelerometers, instead of more expensive piezoelectric analog transducers. The proposed platform provides online filtering and fusion of the collected data directly on-board. Data are transmitted after processing using a WiFi transceiver. Low-cost and synchronized devices permit to have more fine-grained measurements and a comprehensive assessment of the whole building, by evaluating their response to vibrations. The challenge addressed in this paper is to execute a quite computationally-demanding digital filtering on a low-cost microcontroller STM32, and to reduce the signal-to-noise ratio typical of MEMS devices with a spatial redundancy of the sensors. Our work poses the basis for low-cost methods for elaborating complex modal analysis of buildings and structures.
\end{abstract}

Keywords-Structural Health monitoring, SHM, Embedded platform, Distributed measurement.

\section{INTRODUCTION}

The evolution in buildings construction towards the design and realization of increasingly complex structures (i.e. bridges, damns, towers.. ), with new materials and techniques, creates the need of a continuous and autonomous monitoring of the structural integrity of the buildings during the years. Distributed and low cost analysis systems are fundamental even when dealing with old and historical buildings because they are used by people everyday, and many are built in critical regions subjected to potential earthquakes or landslides. Hence evaluating the robustness of infrastructures over the time becomes fundamental for people safety.

The primary function of Structural Heath Monitoring (SHM) is to provide a continuous assessment of structural integrity and promptly identify damages, while generating periodic reports about the state of the structure [1]. In particular, civil engineers need periodic information to assess the behavior of the building over long intervals of time, and the response to the stresses that usually happen on a building (i.e. wheatear, wind, numbers of people inside, ....).

Traditional SHM systems are expensive, because of the high accuracy and sensitivity required by the sensors. Experts are often limited by the cost of such instruments and they deploy a limited number of the sensors in few specific positions of the building. Unfortunately, this methodology cannot produce enough data to understand the dynamic movements and the stability of buildings in a global and comprehensive manner. Moreover, most of the SHM solutions currently on the market are designed for short-term measurement campaigns, because their costs forces experts reuse and share them. This contributes to increasing the final cost of the structural monitoring campaign, because operators need to go periodically every year to install instruments and log new data, for a short period of time.

Recently, distributed and wireless SHM solutions have been proposed on the market [2] to ease the access to the data, but are still characterized by high costs due to expensive sensors, which usually consist of piezoelectric transducers, which have been considered so far as absolutely necessary for acquiring the small vibrations and movements of the building structures with sufficient precision.

In this paper we present a wireless low-cost SHM monitoring system, based on inexpensive MEMS sensors generally used for consumer applications (e.g. in smartphones). We develop a signal processing pipeline that boosts accuracy, achieving a precision comparable to high-end piezoelectric instruments at an order-of-magnitude lower cost. Furthermore, the installation is non-invasive and permits to deploy quickly groups of nodes for a distributed assessment of the whole building. In fact multiple and synchronized measurements in multiple points of the structure allows deeper analyses, such as the identification of modal parameters of the structure, the mechanical frequency of the building when stressed by wind or other external forces, the damping effect, stiffness... Without internal modifications to the structure, the dynamic behavior remains usually unchanged over the time, and minimal drifts over the years are registered. Conversely, when damages occur, the dynamic behavior changes remarkably; and the normal modes of building oscillations are different in frequency, width and number.

Traditional analog monitoring systems consist of singleaxis accurate and expensive piezoelectric accelerometers deployed in specific points of interest and cable-connected to a single monitoring system. The data are then stored and transferred to the processing system. The contribution of this work is to replace a single piezoelectric sensor -single axiswith a redundant configuration of three-axis analog MEMS 
accelerometers. The information is then filtered and processed to achieve high accuracy with 16bit resolution, before sending the information wirelessly over a WiFi channel and collecting all the distributed measurement points with synchronized signals. Each node of the distributed system measures the accelerations of the structure providing components on three orthogonal axes, and thank to the presence of redundant MEMS accelerometers; the achieved SNR is remarkably reduced and comparable to more expensive piezoelectric transducers.

The paper is organized as follows. Section II presents the related works, while Section III describes the building used for our test and the position where the prototypes are installed. Section IV presents the hardware design of the prototype. Discussion about the data pre-processing and filtering are in Section $\mathrm{V}$, together with the firmware organization presented in Section VI. Experimental results are discussed in Section VII, and then we draw our conclusion about the presented work.

\section{RELATED WORK}

Structural health monitoring is based on methodologies that often require expensive instrumentation to provide information with the necessary rate and accuracy. Some methods need accurate measurements done by experts and repeated regularly after a medium-long time interval. For example, the work in [3] used lasers scanners to construct a 3D model of the buildings, that is later compared with a baseline to detect actual alterations of the environment under measurement.

Other methods are more suited for continuous monitoring and permit more frequent evaluation of the building, such as the method described in [4] that permits dynamic analysis of specific civil structures such as bridges or towers [5]. However, these solutions still have a quite high cost of installation due to cabling between logger and sensors, and for data transfer. In fact, such data logging systems for SHM consist of a number of piezoelectric sensors, cabled with the logger. The structure's response is determined by external or internal mechanical excitations such as weather phenomena or wind, vehicle traffic, seismic events, pedestrian action [6], mass movements [7] or to the use of specific excitation hardware like mechanical shakers [8]. In all these cases, the analysis of the response is then calculated offline manually, or through automatic tools that retrieve data directly from a database. Some SHM uses the same piezoelectric sensors, as actuators because already attached to the building and operators can directly tune the intensity of the excitations [9][10].

Recent advances in energy harvesting techniques and in ultra low-power sensing, allowed to design some monitoring systems that can operate virtually forever, such as [11][12], however, the authors have mainly focused on the design of the hardware, without any specific validation of the quality of the data acquired, which is extremely critical especially when using inertial sensors for SHM.

Low-cost SHM devices, with a synchronized logging of the vibrations from different points of the structure is still a challenge goal. Even if wireless sensor networks (WSNs) are a promising technology for such an application, today they cannot maintain the promise of cheap installations. Recent works have deployed similar SHM solutions in tunnels [13][14], or ancient buildings [15] but the costs registered are still too high for commercial uses, in particular because of expensive high precision transducers necessary for the such measurements. In contrast, in this paper we present an inexpensive platform with both reasonable computational power, adequate sensing accuracy, at the cost of few tens of euros.

\section{SETUP}

The building used for our field tests is a tower located in the engineering campus at the University of Bologna, Italy. The tower is used as a library archive and contains more than 60,000 volumes. It has a rectangular footprint of $5 \mathrm{~m} \times 7 \mathrm{~m}$, and is 45 meters high. The structure is characterized by 4 rectangular columns till the top to sustain the building. The experiments are realized using 4 boards installed at different levels of the structure as depicted in Fig. 1. We used 4 sensors to explore how the signal acquisition can be improved by using spatial redundancy, at the various levels of the tower.
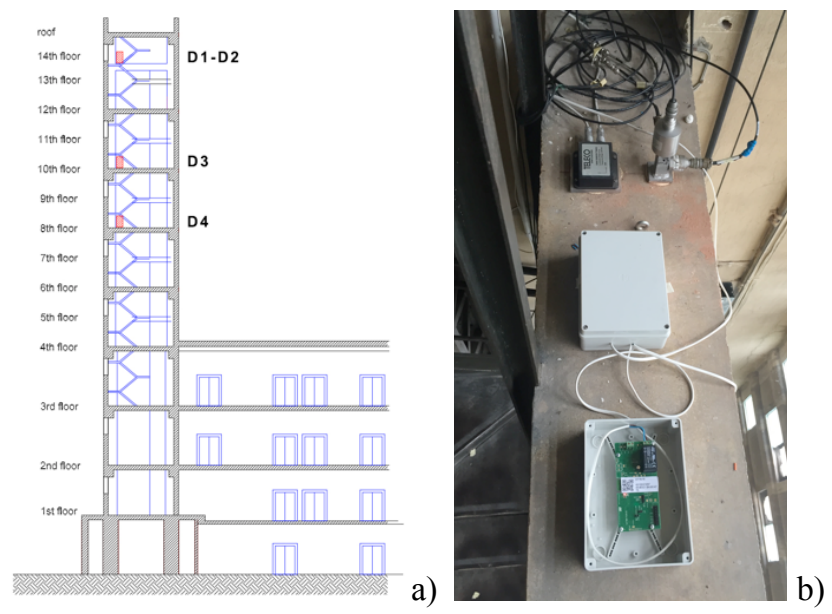

Fig. 1. A) Model of the building with the tower used for the SHM monitoring; b) Deployment of our low-cost solution (bottom) and comparison with a pre-existing piezoelectric monitoring system (top of the figure).

\section{SYSTEM DESCRIPTION}

A distributed network of smart sensors is organized and deployed in specific points the building; communication is point-to-point with the gateway. Data are collected and processed locally on the node. The results of the elaboration are sent through a WiFi module to a NOSQL database in the cloud. Wireless communication has been selected to accommodate the data rate generated by the nodes and because of the availability of $\mathrm{WiFi}$ connectivity in many public/private buildings that permit to reduce the cost of installation in comparison to specialized solutions that require proprietary gateways to forward data on the internet. A schematic overview the organization of the distributed systems is shown in Fig. 2 .

Each wireless sensor node, called DYSP, is based on a microcontroller STM32F405, an ARM Cortex M4 micro- 
architecture used as a central processing unit with SRAM memory of $192 \mathrm{Kbytes}, 1 \mathrm{Mbytes}$ FLASH. It is equipped with a Floating Point Unit FPU, used to implement efficiently the filter stage for collected data. Results are sent through a WiFi module, provided by STMicroelectronics, with dedicated libraries for the wireless protocol stack.

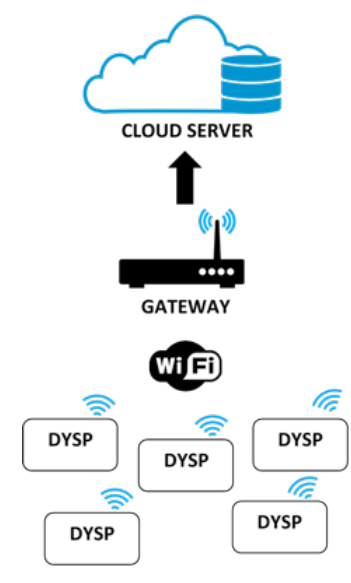

Fig. 2. Organization of distributed monitoring system.

The schematic block of the single node is presented in Fig. 3. Notice that the structural movements and vibrations are measured using a redundant combination of four LIS344ALH, that is a three-axis analog MEMS accelerometers, mounted with $90^{\circ}$ rotation each other. The MEMS accelerometers feature a range of $\pm 2 \mathrm{~g}$ and a maximum bandwidth $1,8 \mathrm{kHz}$. The analog output is connected to a 12 bit $\mathrm{AD}$ converter already available in the MCU. The MEMS accelerometer, used in our prototype, features the lowest acceleration noise density $(50 \mu \mathrm{g}$ $/ \sqrt{ } \mathrm{Hz}$ ) in this class of device; and this was the main parameter that motivated our design choice.

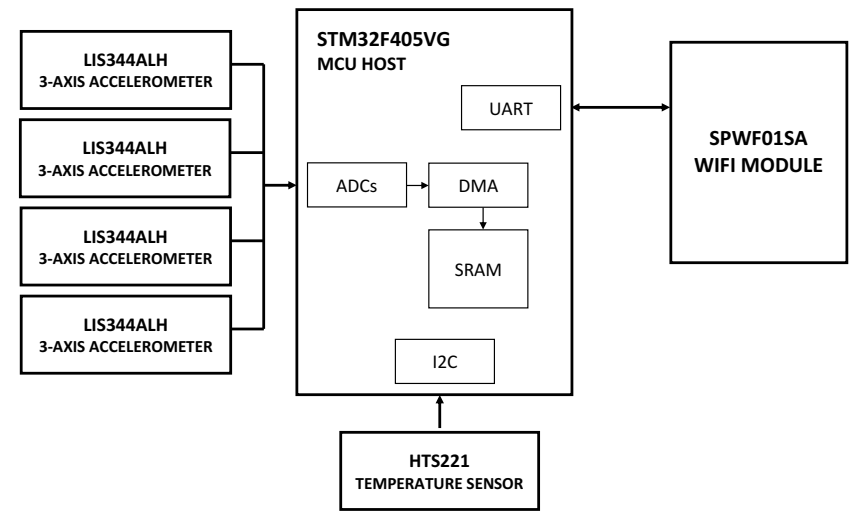

Fig. 3. Schematic block of the board with the four accelerometers.

A drift of the output signal caused by the change in temperature is a typical characteristic of most of the MEMS accelerometers available on the market. We characterized such a drift, as shown in Fig. 4. Notice that the output signal slowly varies during the day, even if the node is steadily mounted in this location. To mitigate this effect, we compensated the thermal drift of the accelerometers thank to the information from a digital temperature/humidity sensor with I2C interface mounted on the same board. Moreover, to compensate offsets that some MEMS may present, we decided a particular displacement of the four sensors, as presented in Fig. 5. The picture shows the board mounted by STMicroelectronics, and highlights position of each accelerometer.

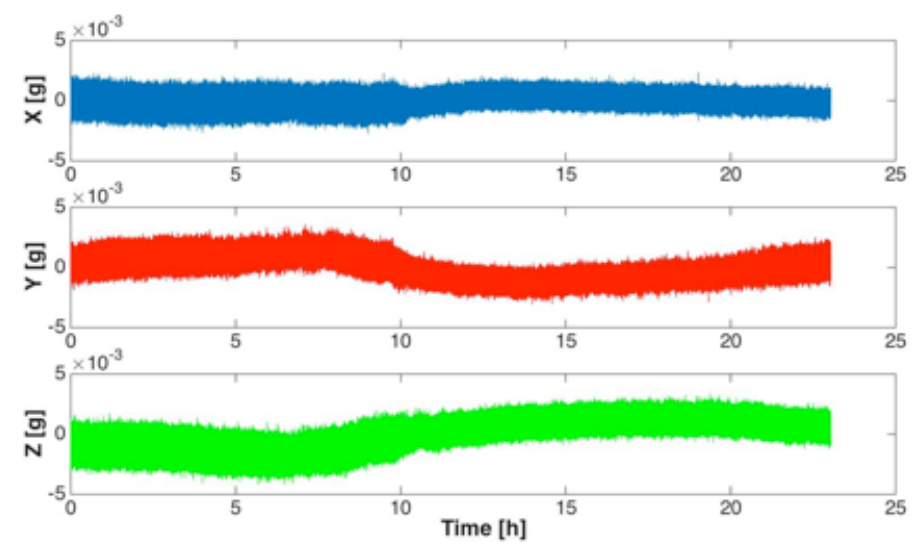

Fig. 4. Example of a full-day acquisition of a node placed in the structure. Data are from a single analog accelerometer on the board. Notice that the signal average oscillates with a daily period, because of the rise of the temperature during the day.

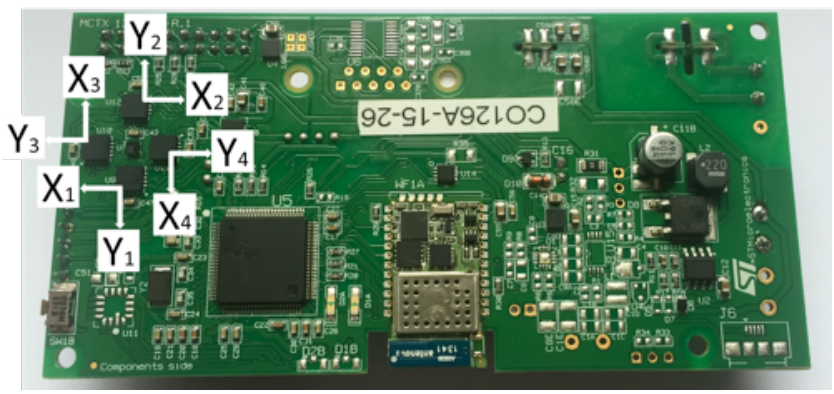

Fig. 5. Picture of the prototyping board with four MEMS accelerometers.

\section{SHM SIGNALS PROCESSING}

Any building is subjected to stresses due to people that everyday use the environments or the weather phenomena. Any stress generates vibrations, similar to micro-seismic signals that crosses the structure and can be measured by the accelerometers. Buildings are usually designed to have lowfrequency normal modes, thus the signals measured by accelerometers are usually in the $0-20 \mathrm{~Hz}$ bandwidth; and the main normal modes of the structure are in the range $1-10 \mathrm{~Hz}$.

When other type of stresses happen and the associated energy changes the integrity of the structure (e.g. earthquakes), the typical frequencies increase and can reach higher range (typically in the bandwidth of $20 \mathrm{~Hz}-45 \mathrm{~Hz}$ ).

We designed the acquisition chain to provide measures at the rate of $100 \mathrm{~Hz}$. To meet the requirements about sensitivity and resolution we oversampled the signals to achieve 16bit 
resolution from the internal 12 bit $\mathrm{ADC}$ available on the STM32F405 microcontroller. Oversampling improves the signal to noise ratio of quantization (SQNR) and consequently increases the ADC resolution. To approach the performance of expensive piezoelectric transducers, we extended the conversion resolution with additional 4bits, by oversampling the signal 256 times, the final desired output rate. Thus the final sampling frequency is $f o s=25,6 \mathrm{kHz}$ for each axis of the accelerometers. A further advantage associated to oversampling the signal is that the quantization error is distributed to a larger range of frequencies, which ease the implementation of digital filters. The digital filter is also designed to decimate the final data rate to the requirement of $100 \mathrm{~Hz}$. The acquisition chain is described in Fig. 6.

As previously mentioned the signal from the accelerometers is acquired with the ADC at a frequency of $25,6 \mathrm{kHz}$. Data transfer is organized using the DMA from the ADC to the memory. This permits to achieve a regular and fast data transfer, while the CPU is less loaded and available for other activities. In addition, the DMA is used with a double buffer configuration. When the DMA writes the acquired data to a buffer, the CPU processes a second buffer already filled by the DMA in the previous cycle.

The four accelerometers are simultaneously sampled and data are organized according to the orthogonal directions. Values are subsequently averaged, for compensating spatial deviations thank to redundancy. After this processing stage, data from each axis is then filtered using a low-pass filter with $50 \mathrm{~Hz}$ cutoff frequency. The filter is implemented using polyphase IIR Butterworth configuration, consisting of 8 stages, each of them with more selective cutoff frequency. In each stage, we also decimate the output results until the final required data rate of $100 \mathrm{~Hz}$. For this, we process 256 samples every $10 \mathrm{~ms}$ time step.

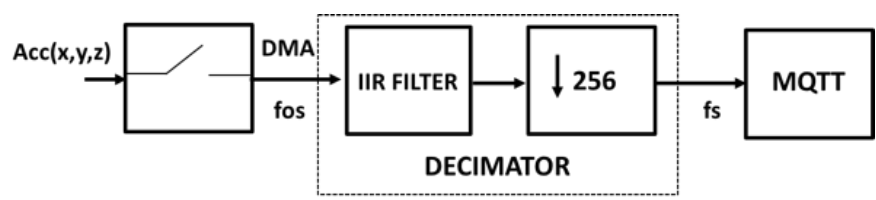

Fig. 6. Acquisition chain consisting in sampling, conversion, IIR filter and decimation.

\section{FIRMWARE ARCHITECTURE}

The firmware implemented in the DYSP executes on the top of FreeRTOS, a real-time operating system for microcontroller-based embedded systems. FreeRTOS is open source and permits to separate the acquisition/transmission chain in different independent tasks that can share resource and peripherals.

The DYSP is regulated with four tasks:

i) the ModalStreamingTask, that is responsible for receiving the samples acquired by the DMA, filtering and storing thee results in a buffer with 10Ksamples (called MODAL_BUFFER). The choice of the size depends on the WiFi streaming capability. The optimal configuration for WiFi transmission is to send continuously 100 s of measurements (sampled at $100 \mathrm{~Hz}$ ).

A synchronization method, implemented using a binary semaphore, is used to notify to the ModalStreamingTask about the completion of the data transfer to current one out of two buffers used in double-configuration. Whenever the DMA transfer completes a buffer with 256 samples, the semaphore is released and triggers the ModalStreamingTask. The IIR filter provides new data for each axis at $10 \mathrm{~ms}$ rate, and appends the result in the MODAL_BUFFER. When the buffer is completed with 100 s recordings, it is transferred to a communication task called NetworkConsoleTask, using a thread-safe FIFO defined by freeRTOS, for transmitting the information to the wireless module. The decision to split the workload into two tasks is driven by the sampling and processing activity that requires time critical DMA operations. In this way, data transmission data is separated in an independent task and the ModalStreamingTask is optimized for processing activities and data losses are limited as much as possible.

The ii) NetworkConsoleTask manages the communication channel. Data queues are provided for data reception and transmission; while the task initializes the MQTT protocol to establish a connection to the broker (MQTT server) and send the data.

The message payload is formatted using a JSON object with timestamp and data. The format is decided to facilitate the packet processing at server-side data management. Robust control and recovery routines permits to reestablish a connection if dropped. In fact, due to the unstable nature of the WiFi connection, it may occur that the MQTT link closes abruptly without saving internal status.

A third task, called iii) WebListenerTask will monitor the MQTT connection and reestablish it in case of disconnections.

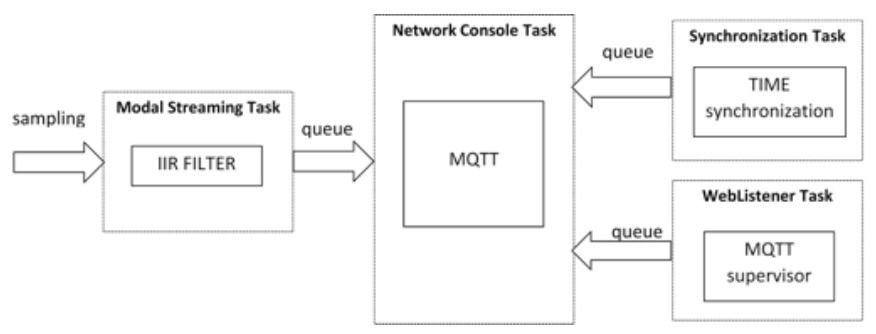

Fig. 7. Organization of the tasks and their dependencies.

The last critical feature for a distributed SHM, is time synchronization. In fact, considering multiple points of measurement, an accurate synchronization for the timestamps measured in different position of the nodes deployment is a strict requirement. This determines the correlation needed for calculating a complete modal analysis of the whole building.

A last task, called iv) SynchronizationTask is designed to precisely manage the temporal synchronization between the DYSP and to check the time deviations between a local realtime clock (RTC) in the microcontroller and the timestamp provided by a NTP server used as a reference. The synchronization algorithm implemented on board permits to 
achieve precision in assigning the timestamp to the samples below $<1 \mathrm{~ms}$; for each DYSP belonging to the same network.

\section{EXPERIMENTAL RESULTS}

The measurement setup consists of four DYSP nodes placed nearby a preexisting commercial SHM system [2], that was used to measure the modal frequencies of the building. Thus the dynamic behavior of the structure and its model is already well-know and studied. The goal of our test is to verify that similar analysis can be calculated with the signals measured by our distributed monitoring system whose cost is one order of magnitude lower. A WiFi router was positioned at the top of the tower, connected to the campus LAN network.

From pre-existing studies, the main modal frequencies of the building is calculated at $1,5 \mathrm{~Hz}$ and a secondary mode was identified at $3 \mathrm{~Hz}$. This elaboration was carried out using a conventional monitoring system equipped with single axis piezoelectric accelerometers, a setup confirmed by experts in civil engineering and recommended for the use of the preexisting commercial system.

The measurements and the analysis done with data acquired with the proposed system concern only to the $\mathrm{X}$ and $\mathrm{Y}$ axes of the DYSP, as the Z-axis perpendicular to the ground is negligible in our case, given the rigid nature of the structure. Moreover, no previous data are available for this direction because of the single-axis transducers used by the conventional measurements. Moreover, due to the total absence of urban traffic nearby, the only excitations for the building are generated by the wind and the people working inside, and these stresses generates only responses in $\mathrm{X}$ and $\mathrm{Y}$ directions.

The dynamic behavior of a structure, in terms of natural frequencies, damping and mode shapes, is described by means of the power spectral density function (PSD) that contains all the information needed by the experts. In fact, one can determine the vibration modes through the PSD amplitudes, while the damping can be assessed by measuring the width of the peaks of major frequency components (i.e. @ $1,5 \mathrm{~Hz}$ and (a) $3 \mathrm{~Hz}$ in our case).

A first set of measurements was carried out using measures from each single accelerometer available on the DYSP. Then we evaluated the improvements of the SNR, when the signals from all the MEMS sensors mounted in spatial redundancy, are averaged according to the main directions.

Fig. 8 shows an example of the data acquired in a whole day from the four devices according to the same direction (X direction). Notice the presence of a fluctuation in the acceleration, for all the three axes, due to the temperature and noise. For the sake of clarity, and to make the plot readable, we shifted all the traces with a fixed offset; while the central trace (green) is obtained by averaging the signals from the four sensors. The noise is remarkably reduced thank to the average and the filtering stage, that improves the signal-to-noise ratio.

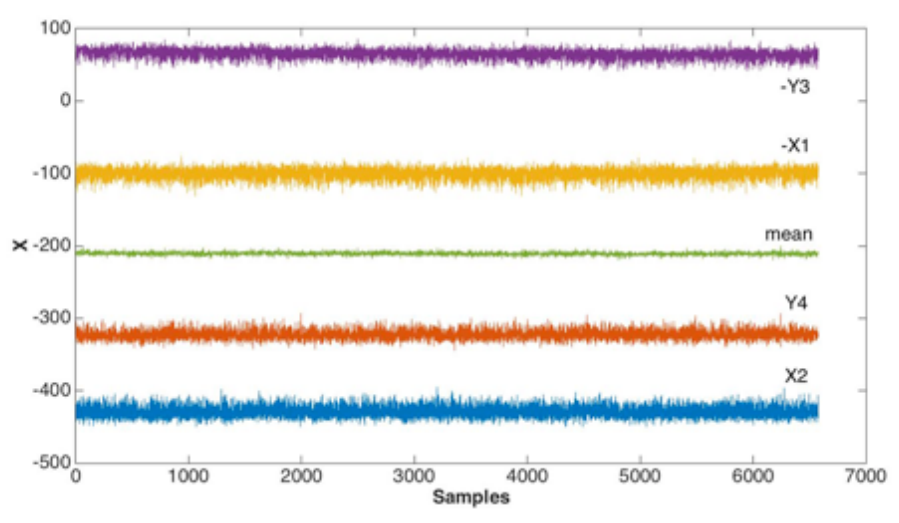

Fig. 8. Comparison between the signals from the four accelerometers and their average using the axes in the same direction. The noise is much more attenuated, and temperature drift is compensated.

Fig. 9 shows the PSD of the signal acquired by a single MEMS accelerometer using the DYSP. Notice that the frequency components at $1,5 \mathrm{~Hz}$ is visible as expected for the $\mathrm{Y}$ axis, but it not recognizable for the $\mathrm{X}$ axis component. Moreover the second modal component at $3 \mathrm{~Hz}$ is not evaluable as well, for both orientation, due to the noise associated to the measurement.

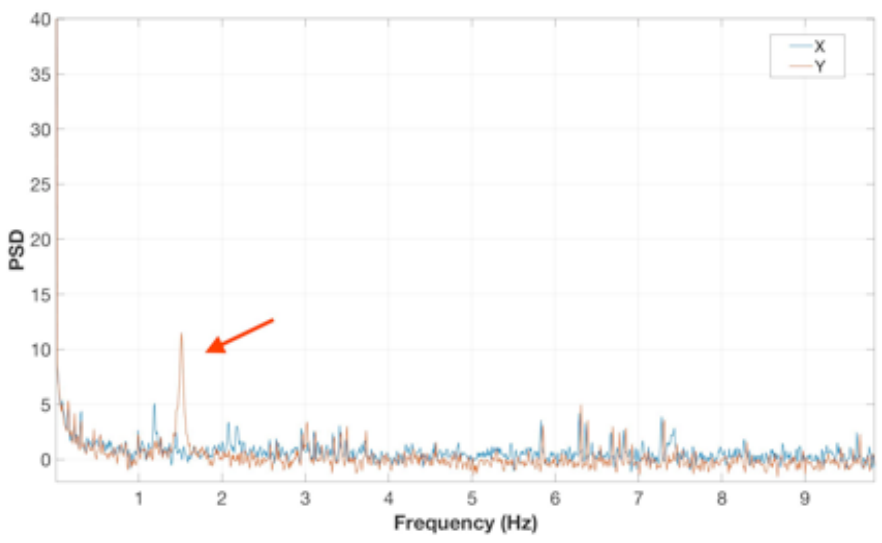

Fig. 9. PSD analysis on two axis using the data from a single accelerometer on the DYSP prototype.

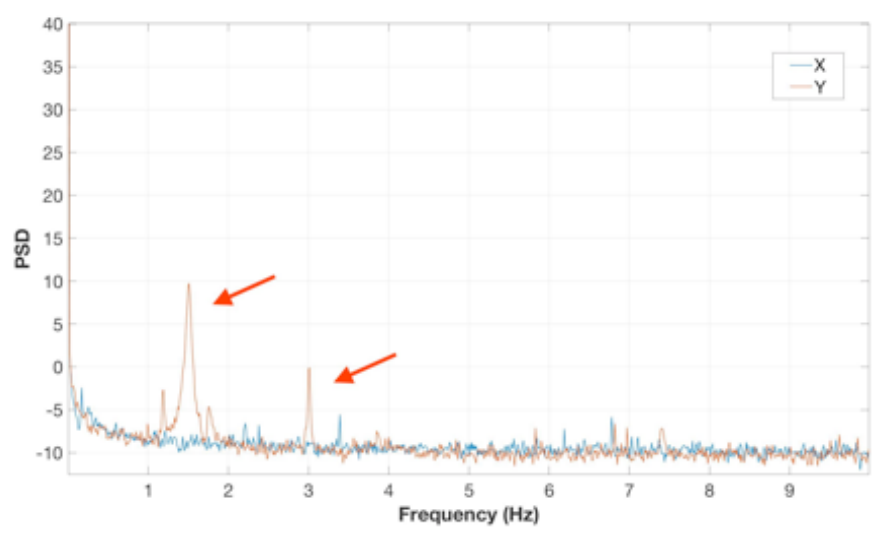

Fig. 10. PSD analysis on two axis of the averaged signal from the redundant configuration. With a redundant configuration, the detection of a second normal mode, typical of the structure is now possible. 
Fig. 10 demonstrates the effectiveness of the proposed approach. The PSD is calculated with the same method and with the same setup, while the data used for the SHM evaluation are provided by the average of the four accelerometers in spatial redundancy on each single DYSP. In this elaboration, the principal modal frequency is still visible as in the previous one. Now, also the secondary modal frequency of $3 \mathrm{~Hz}$ is clear and adequate for modal analysis and SHM assessments.

\section{CONCLUSION}

Structural Health Monitoring (SHM) solutions are gaining interest in the engineering and construction communities, because can provide reliable and fast feedback in case of alterations of the buildings. The cost is usually a major concern that limit the capability of measurement points and limit the possibility of such application. We presented a low-cost wireless device for SHM, which leverage on the low-cost of the sensors, i.e. 10x less of the classical piezoelectric devices, the possibility to create a large distributed application with multiple points. The application provides and efficient online pre-processing on the data acquired, reducing the SNR ratio and provides data that civil engineers can directly use for realtime assessment of the building.

\section{ACKNOWLEDGMENT}

The research contribution presented in this paper has been supported by the EuroCPS Project (grant $n$. 644090) and by the FLEXMETER Project (grant n. 646568), both funded by the EU H2020. Moreover, the research has been funded by a research grant of STMicroelectronics that provided MEMS and devices.

\section{REFERENCES}

[1] Farrar, C.R., Worden, K., An introduction to structural health monitoring, (2007) Philosophical Transactions of the Royal Society A Mathematical, Physical and Engineering Sciences, 365 (1851), pp. 303315 .

[2] Roberto Guidorzi, Roberto Diversi, Loris Vincenzi, Claudio Mazzotti, Vittorio Simioli, Structural monitoring of a tower by means of MEMSbased sensing and enhanced autoregressive models, European Journal of Control, Volume 20, Issue 1, January 2014, Pages 4-13,

[3] R. Marani, M. Nitti, E. Stella and T. D'Orazio, "Monitoring of indoor environments by change detection in point clouds," 2016 IEEE Workshop on Environmental, Energy, and Structural Monitoring Systems (EESMS), Bari, 2016, pp. 1-6.

[4] A. Bonelli, O. Bursi, R. Ceravolo, S. Santini, and N. Tondini, Dynamic identification and structural health monitoring of a twin deck curved cable-stayed footbridge: the "Ponte del Mare" of Pescara in Italy, in Proc. of the V European Conference on Structural Health Monitoring, Sorrento, Italy, 2010.

[5] R. Guidorzi, R. Diversi, L. Vincenzi and V. Simioli, "AR+ noise versus AR and ARMA models in SHM-oriented identification," 2015 23rd Mediterranean Conference on Control and Automation (MED), Torremolinos, 2015, pp. 809-814.

[6] A. Occhiuzzi, M. Spizzuoco, and F. Ricciardelli, Loading models and response control of footbridges excited by running pedestrians, Structural Control and Health Monitoring, Special Issue: Selected papers from the research project VinCES, vol. 15, no. 3, pp. 349-368, 2008.

[7] L. Vincenzi, C. Mazzotti, and M. Savoia, Modal identification of a TAV viaduct using subspace models, 2nd International FIB Congress, Naples, Italy, 2006
[8] C. Mazzotti, and L. Vincenzi, Structural identification of a steel structure by forced vibrations, ICOSSAR - International conference of structural safety and reliability, Rome, Italy, 2005.

[9] D. Musiani, K. Lin and T. S. Rosing, "Active Sensing Platform for Wireless Structural Health Monitoring," 2007 6th International Symposium on Information Processing in Sensor Networks, Cambridge, MA, 2007, pp. 390-399.

[10] D. Dondi, A. Di Pompeo, C. Tenti and T. Šimunic Rosing, "Shimmer: A wireless harvesting embedded system for active ultrasonic Structural Health Monitoring," 2010 IEEE Sensors, Kona, HI, 2010, pp. 2325 2328.

[11] A. Cammarano, D. Spenza and C. Petrioli, "Energy-harvesting WSNs for structural health monitoring of underground train tunnels," 2013 IEEE Conference on Computer Communications Workshops (INFOCOM WKSHPS), Turin, 2013, pp. 75-76.

[12] D. Boyle, M. Magno, B. O'Flynn, D. Brunelli, E. Popovici and L. Benini, "Towards persistent structural health monitoring through sustainable wireless sensor networks," 2011 Seventh International Conference on Intelligent Sensors, Sensor Networks and Information Processing, Adelaide, SA, 2011, pp. 323-328.

[13] F. Stajano, N. Hoult, I. Wassell, P. Bennett, C. Middleton, and K. Soga, "Smart bridges, smart tunnels: Transforming wireless sensor networks from research prototypes into robust engineering infrastructure," Ad Hoc Networks, vol. 8, no. 8, pp. $872-888,2010$.

[14] L. Mottola, G. P. Picco, M. Ceriotti, c. Guna, and A. L. Murphy, "Not all wireless sensor networks are created equal: A comparative study on tunnels," ACM Trans. Sen. Netw., vol. 7, no. 2, pp. 15:1-15:33, Sep. 2010 .

[15] M. Ceriotti et al., "Monitoring heritage buildings with wireless sensor networks: The Torre Aquila deployment," 2009 International Conference on Information Processing in Sensor Networks, San Francisco, CA, 2009, pp. 277-288. 